\title{
AN INTEGRABLE OPTICS LENS FOR THE PSR AND SNS*
}

\author{
V.Danilov, J.Galambos, D.Jeon, J.Holmes, D.Olsen, ORNL SNS Project, Oak Ridge, TN \\ D.Fitzgerald, R.Macek, M.Plum, LANL LANSCE, Los Alamos, NM
}

\section{Abstract}

This is a study to increase the threshold of the Los Alamos LANSCE Proton Storage Ring (PSR) to the electron-proton (e-p) instability. The approach is to use a solenoid lens system to increase the Landau damping and betatron tune spread, without producing betatron resonances or energy spread that would necessitate increased aperture. This study rests upon the concept of integrable optics, which is a branch of Hamiltonian dynamics that determines how to modify dynamic systems to obtain integrable Hamiltonians having regular phase space maps and no chaos. For such cases, the particle trajectories, when integrated as functions of time and initial conditions, follow invariant surfaces. In essence, the maps are similar to linear maps with the addition of a betatron tune spread; however, the magnetic fields required to obtain such maps are complicated.

\section{INTRODUCTION}

Prior to the recent upgrade program, the average beam current in the PSR was limited to $70 \mu \mathrm{A}$, at $20 \mathrm{~Hz}$ and $2 \times 10^{13}$ protons/pulse. At this intensity, the threshold for the PSR e-p instability limits further current [1]. The PSR e-p instability limits the performance of the LANSCE facility and provides a fundamental uncertainty and risk to the performance of a new spallation neutron source such as the SNS Project.

One of the potential explanations of this instability is the large electron charge in the vacuum chamber, produced by multipactoring and other effects. It has been found [1] that increasing the betatron tune spread using octupoles increases the instability threshold. In particular, increasing the betatron tune spread by about 0.01 could increase the threshold by about a factor of three. Unfortunately, octupoles excite nonlinear resonances that lead to an uncontrolled beam loss. Even so, this is an important result because it shows that modifying the magnetic fields to increase the tune spread can raise the instability threshold. Increasing the energy spread can also increase the tune spread in the PSR; however, this is not a viable solution because of the limited horizontal aperture. We propose to use a special nonlinear element to increase the betatron tune spread without introducing nonlinear resonances. We call such a system "integrable", though in general, phase space from integrable systems can contain resonances.

\section{INTEGRABLE OPTICS}

One particularly simple class of integrable optics applies to axially symmetric magnetic fields. Under certain conditions, solenoid lenses can be used to construct these integrable maps for circular rings. To apply this to the PSR, the lattice must meet two conditions: (1) the solenoid lenses must be installed in a straight section at a location with $\beta_{x}=\beta_{y}$, so that a beam with equal emittances is round; and (2) the fractional tunes of the ring in both planes, without the solenoids, must be either 0.25 or 0.75 . Once these conditions are satisfied the angular momentum, $M=x \cdot p_{y}-y \cdot p_{x}$, an integral of the motion, will be conserved by the axial symmetry of the system. $M$ will also be conserved in the linear storage ring structure.

There is an additional invariant of motion, which is conserved for all values of the angular momentum whenever the nonlinear kick is axially symmetric and the change in radial momentum $p_{r}$ is given by $\Delta p_{r}=-b \cdot r /\left(1+a \cdot r^{2}\right)$, where a and $\mathrm{b}$ are free parameters [2]. Throughout, the normalised variables, $x_{n}=x / \sqrt{\beta_{x}}, y_{n}=y / \sqrt{\beta_{y}}$, are used along with the same normalization for radial variables. If the linear transfer matrices for one turn around the ring for both horizontal and vertical normalised variables are given by $\left(\begin{array}{cc}0 & 1 \\ -1 & 0\end{array}\right)$, then, the invariant after the above lens is:

$\mathrm{I}\left(r_{n}, p_{r}\right)=\left(a \cdot r_{n}^{2}+1\right) \cdot\left(r_{n}+p_{r}\right)^{2}+b \cdot r_{n} \cdot\left(r_{n}+p_{r}\right)+\left(r_{n}^{2}+M^{2} / r_{n}^{2}\right)$.

Figure 1 plots the invariant surfaces given by Eq. 1. In order to model the PSR with an aperture of about $5 \mathrm{~cm}$, the $\mathrm{x}$ coordinate unit scale in Fig. 1 is $10 \mathrm{~cm}$. For this calculation the angular momentum is equal to zero and the coefficients in scaled units are $a=-0.40$ and $b=$ 1.17. The reference orbit betatron fractional tune for both planes is 0.35 with the solenoid present.

The stable motion is bounded by two separatrices, which occur at the points:

$\left(-\sqrt{(b-2) / 2 a},-2 \sqrt{(b-2) / 2 a}\right.$ ) and $\sqrt{(b-2) / 2 a^{2} \sqrt{(b-2) / 2 a}}$. 


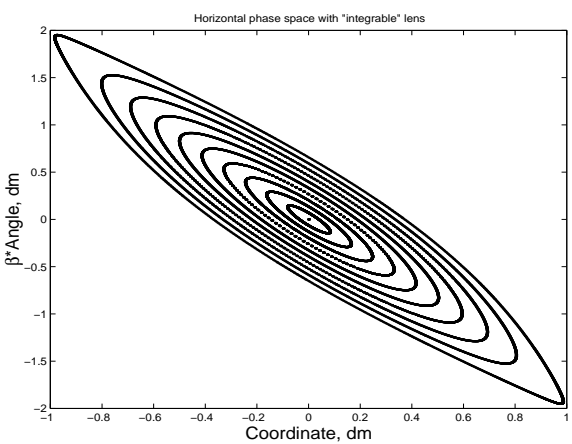

Fig. 1 Phase space motion in the integrable cell. The contours represent motion with initial conditions from the closed orbit up to the limit of the dynamic aperture.

These two points are critical points of the Eq.1 invariant and define the region of stable phase space. If, for example, the beam occupies half the aperture, then the tune spread is found numerically to be 0.006 and is close to what is required. This increase in tune spread is produced without exciting any betatron resonances. If we take into account only the octupole term, the formula for tune spread is: $\Delta v=3 /(8 \cdot 2 \cdot \pi) \cdot b \cdot a \cdot \varepsilon_{0}=6 \cdot 10^{-3}$ for emittance $\varepsilon_{0} \approx 20 \cdot 10^{-6} \mathrm{~m} \cdot \mathrm{rad}$.

We must form the lenses using an axially symmetric magnetic structure in order to preserve angular momentum invariance. All the fields in such a system can be calculated from a single function $\mathrm{H}(\mathrm{s})$, the longitudinal magnetic field on the axis of the lens, which corresponds to the reference orbit. The longitudinal magnetic field in all space is then [3]:

$H(s, r)=\sum_{n=0}^{\infty} \frac{(-1)^{n}}{(n !)^{2}} \cdot H^{(2 n)}(s) \cdot(r / 2)^{2 n}$,

where $\mathrm{r}$ is the radius from the axis, $\mathrm{s}$ is the longitudinal coordinate, and the superscript (2n) denotes the $2 n$-th derivative of $\mathrm{H}(\mathrm{s})$ with respect to the coordinate $\mathrm{s}$. Calculation of nonlinear kicks through third order from such a lens was made in [4]. Here the exact expressions for the nonlinear kick will be used.

The radial and azimuthal motion of a particle can be described by the two simple equations:

$r^{\prime \prime}-r \theta^{\prime}\left(\theta^{\prime}+e \cdot H_{S} / p\right)=0, \quad \theta^{\prime}=\theta_{0}^{\prime} \cdot r_{0}^{2} / r^{2}-e \cdot \Phi /\left(2 p \pi r^{2}\right)$,

where the derivatives are taken on the longitudinal coordinate, and $\theta, \theta_{0}$ are the azimuthal and initial azimuthal angle of the particle, and the same notation is used for the radius $r$. The symbol $\Phi$ stands for the magnetic flux and is equal to $\Phi=2 \pi \int_{0}^{r} H_{s} \cdot r^{\prime} d r^{\prime}$, where $H_{S}$ is taken from Eq. 3. Finally, Eqs. 4 can be combined to yield:

$$
\begin{aligned}
r^{\prime \prime}-r \cdot\left(\theta_{0} \cdot r^{2} / r^{2}\right) & \stackrel{2}{=} \frac{e}{p} \cdot \theta_{0} \cdot r^{2} / r^{2}\left(\frac{\Phi(r)}{\pi r^{2}}-H_{S}(r)\right)+ \\
& \frac{e^{2}}{(2 \pi p)^{2} r^{3}} \cdot\left(\Phi(r)^{2}-\Phi(r) \cdot \Phi(r)_{r}^{\prime} \cdot r\right) .
\end{aligned}
$$

The force term on the L.H.S. of Eq. 5 is just the centripetal force, which always appears in cylindrical coordinates. The first term on the R.H.S. is a linear function of the longitudinal field derivatives, and its integral is zero because the function at the ends is zero. Consequently, only the last term of the R.H.S. is of interest, and is denoted by:

$$
\begin{aligned}
K(r, s)= & \frac{e^{2}}{p^{2}}\left(-\frac{1}{4}\left(H^{(0)}\right)^{2} \cdot r+\frac{1}{8} \cdot H^{(0)} \cdot H^{(2)} \cdot r^{3}-\left(\frac{1}{128} \cdot H^{(0)} \cdot H^{(4)}\right) \cdot r^{5}+\right. \\
& \left.\left.\frac{3}{256} \cdot\left(H^{(2)}\right)^{2}\right) \cdot r^{5}+\left(\frac{1}{4608} \cdot H^{(0)} \cdot H^{(6)}+\frac{1}{768} \cdot H^{(2)} \cdot H^{(4)}\right) \cdot r^{7}-. .\right)
\end{aligned}
$$

where $H^{(n)}$ is the n-th derivative of the longitudinal field on the axis.

The radial kick can be obtained by integrating $K(r, s)$ over $\mathrm{s}$, regarding the radius $\mathrm{r}$ as a constant. The integration yields, where we ignore the centripetal force and consider only the kick from the magnetic field:

$$
\begin{aligned}
\Delta r^{\prime} & =\frac{e^{2}}{p^{2}}\left(-\frac{r}{4} \int_{-\infty}^{\infty}\left(H^{(0)}\right)^{2} d s-\frac{r^{3}}{8} \int_{-\infty}^{\infty}\left(H^{(1)}\right)^{2} d s\right. \\
& \left.-\frac{5 \cdot r^{5}}{256} \int_{-\infty}^{\infty}\left(H^{(2)}\right)^{2} d s-\frac{7 \cdot r^{7}}{4608} \int_{-\infty}^{\infty}\left(H^{(3)}\right)^{2} d s-\ldots\right)
\end{aligned}
$$

\subsection{Physics Design of Axially Symmetric Integrable Lens from Solenoids}

Now let us construct the integrable lens from one-wire coils. The magnetic field from a one-wire coil on the symmetry axis is:

$H[$ Tesla $]=2 \pi 10^{-7} I[$ Amp $] \cdot R^{2} /\left(R^{2}+s^{2}\right)^{3 / 2}$,

where $R$ is the radius of the coil, $I$ is the current, and $s$ is the longitudinal coordinate with respect to the coil. It has found numerically that two coils with the same longitudinal coordinate dependence can be utilised to produce the needed nonlinear lens with very good accuracy. A ninth order Taylor expansion of the kick on the radial coordinate found relative coil radii of $R$ and 
$1.5 R$, and relative currents of $I$ and $-0.65 I$, respectively. Fig. 2 shows a schematic view of this lens.

Now let us write the parameters of this lens in terms of the inner radius $R$ and current $I$. The radial angle change is:

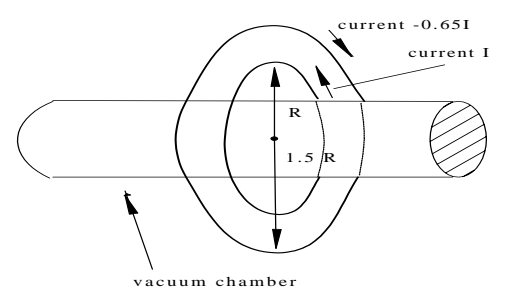

Figure 2: Schematic view of nonlinear lens

$\Delta^{\prime}=\frac{R^{2} \cdot 0.1}{r_{L}^{2}}\left(-0.73 \frac{r}{R}-0.64 \frac{r^{3}}{R^{3}}-0.6 \frac{r^{5}}{R^{5}}-0.55 \frac{r^{7}}{R^{7}}-0.5 \frac{r^{9}}{R^{9}}\right)$,

where $r_{L}(m)$ is the Larmour radius corresponding to the maximum magnetic field at the axis. The ratio of all successive pairs of coefficients is nearly constant, and is equal to $0.90 \pm 0.02$. This provides the value of $a$ for the kick: $a=-0.9 \cdot \beta / R^{2}$. The coefficient $b$ for the same kick is $0.073 \cdot R \cdot \beta / r_{L}^{2}$.

To obtain a linear shift from this lens the coefficient $b$, as shown in Fig. 1, must be 1.17. Substituting the parameter values $R=0.05 \mathrm{~m}, \beta=10 \mathrm{~m}$ appropriate to the PSR, we obtain $r_{L}=0.2 \mathrm{~m}, D \approx 5 \mathrm{~cm}$ for the Larmour radius and dynamic aperture, respectively, and the coil current is:

$$
I(\mathrm{~A})=\frac{v}{c} \cdot E(\mathrm{GeV}) \cdot R(\mathrm{~m}) / 0.6 \pi \cdot 10^{-7} \cdot r_{L}(m) \approx 2 \cdot 10^{6} \mathrm{~A} .
$$

Clearly such a solenoid with normal conducting cu coils would require many turns. When many turns are used with a reasonable current density it is impossible to design a coil producing the correct end field shape to serve as an axially symmetric integrable-optics lens.

A superconducting variation of this idea may be more feasible. Consider a 10-coil variant with an optimal coil aspect ratio found by numerical optimisation. The current in the coil is taken to be 600 kA. Additionally, the inclusion of iron provides a $65 \%$ increase in the cubic nonlinearity compared to the case with no iron. Figure 3 shows the coil configuration used to model the impact of the iron inserts on the field as calculated with Poisson. Contours of constant field level are also shown. The iron encompasses all the coils and extends up to $13 \mathrm{~cm}$ from the axis, the white contour delineates the positive current area $(600 \mathrm{kA})$ and the black contours delineate the negative current (-600 kA) area. The fields and current density can be achieved with $\mathrm{NbTi}$ cable. A more detailed description of the calculations can be found in Ref. [5]. The betatron tune spread for this case is 0.004 , which is close that needed. A more expensive superconducting variant could use lower temperatures and higher fields to permit operation at up to $14 \mathrm{~T}$, which would provide a tune spread four times higher. Another variant is a pulsed lens with the magnetic field up to 20 Tesla.

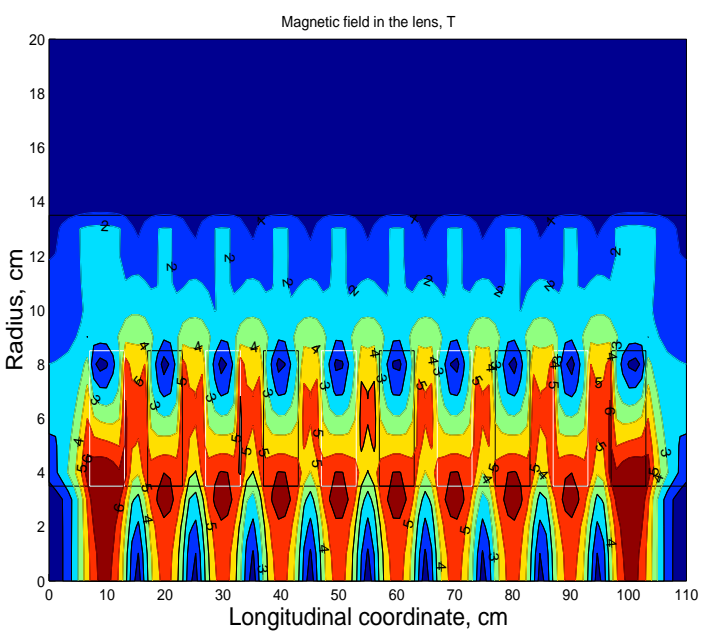

Fig. 3. Magnetic field in superconducting solenoid

\section{CONCLUSIONS}

The use of a solenoid lens to increase the betatron tune spread, and hence Landau damping and the threshold of the LANSCE PSR instability has been studied. Such a lens could increase the threshold without producing betatron resonances or energy spread that would require increased aperture. It is found that such a lens could be fabricated, but the required field shape and intensity would require the use of superconducting cable.

\section{ACKNOWLEDGMENT}

*Research on the Spallation Neutron Source is sponsored by the Division of Materials Science, U.S. Department of Energy, under contract number DE-AC0596OR22464 with Lockheed Martin Energy Research Corporation for Oak Ridge National Laboratory.

\section{REFERENCES}

[1] D. Neufer, et al., Particle Accelerators 23, 133-148 (1988).

[2] V. Danilov, E. Perevedentsev in PAC 97, 1997

[3] V.V. Batygin, I.N. Toptygin, in Problems in Electrodynamics, ed. By D. ter Haar (Academic Press, London, 1978), p.279.

[4] V. Danilov et al. "Dynamic Aperture Limitations in Storage Rings Due to Solenoids", EPAC 1990, Nice, France

[5] V.Danilov et al. "Proposal for Solenoid Lens Experiment at PSR", SNS Tech Memo, March, 1999 